\title{
What the probation officer expects from the psychiatrist
}

\author{
John Harding \& Angus Cameron
}

The origins of the probation service can be traced back to the late-Victorian era and the introduction of the so-called police court missionaries who supervised offenders on conditional release from the court. The service grew slowly, but mushroomed in the 1970 s and 1980s when several major acts widened the scope of the service by introducing parole, aftercare of discharged prisoners, community service by offenders, and extended the scope of probation and bail hostels. Since the beginning of this decade the probation service has been working in an everchanging legal and philosophical context. Public protection and the prevention of crime are our primary aims. Legislation and ministerial directives have necessitated increasing involvement of probation offices in work with offenders with mental disorder, and have brought into greater focus the relationship of the probation service with psychiatry.

This article attempts to illustrate some of the tensions at the interface of psychiatry and the criminal justice system as experienced by the Inner London Probation Service (ILPS). Both the health service and the probation service are again facing major changes in terms of proposed criminal justice and mental health legislation. The Government's strategies for both services will emphasise attention to protection of the public and assessment of risk, practice that is evidence-based, and working across traditional boundaries. What the probation service wants from psychiatry is a working partnership to develop timely and defensible interventions with offenders with mental disorder, allowing each service to assist the other in the achievement of their strategic goals.
This article concludes with a number of action points for developing cooperation between psychiatric and probation services.

\section{A case vignette}

The following vignette is a composite of actual cases, with details altered for illustrative purposes.

$\mathrm{Mr} \mathrm{A}$ is a young man with no known personal or family history of mental illness. Developmentally normal, he was unruly at school, leading finally to expulsion. A series of court appearances followed, with several convictions for crimes involving theft.

Mr A was deemed mentally unwell while serving his most recent prison sentence. He was transferred to hospital under Section 47/49 of the Mental Health Act 1983 (MHA) as being in immediate need of treatment and was diagnosed as suffering from acute psychosis. His treatment was haloperidol $50 \mathrm{mg}$ three times a day in addition to injections of clopixol $200 \mathrm{mg}$ fortnightly. He was later additionally prescribed lithium carbonate $250 \mathrm{mg}$ three times a day. On admission to hospital he was observed to be overactive and elated, speaking rapidly and expressing grandiose delusions. He was negligent of his personal hygiene and was verbally and physically aggressive. Although there was improvement in his mental state, his aggressive and assaultative behaviour continued throughout his stay in hospital and eventually the responsible medical officer (RMO) decided that his behaviour

John Harding is Chief Probation Officer of the Inner London Probation Service (ILPS, 7-73 Great Peter Street, London, SW1P 2BN). He was a founding commissioner of the Wessex Project in Hampshire. He has contributed widely in books and articles on criminal justice, is a visiting expert at the United Nations Far Eastern Crime Prevention Institute in Tokyo, an adviser to the International Police and Crime Studies at Portsmouth University and an Associate Fellow of the Manheim Centre at the London School of Economics. Angus Cameron originally joined the ILPS in June 1975. Since 1996 he has been the Mental Health Advisor to ILPS where he is the Senior Probation Officer with responsibility for offenders with mental disorder. He has contributed to a number of publications on provision of service and management of offenders with mental disorder. 
could not be attributed to the presence of mental illness and that it was consistent with presence of a psychopathic personality disorder.

He was returned to prison shortly before his release date. He remained in receipt of antipsychotic medication during his remaining stay in prison. The prison was remote from his home area and the treating psychiatrist referred him to his catchment area forensic services for continuing care. No service was in place by his release date and he was discharged to his parent's home with a referral to his family general practitioner (GP) to administer his medication. He was discharged on flupenthixol $200 \mathrm{mg}$ fortnightly.

The discharge plan, for him to stay at home, quickly broke down and he was placed in a local hostel out of his GP's catchment area. The hostel had no GP cover at that stage and the family GP refused to continue administration of the medication. At the hostel, he became hostile to staff and talked about setting fire to the hostel. The probation service and hostel staff were unable to engage any of the community health or social services to carry out an assessment. The probation officer had written to the forensic services asking the community forensic psychiatrist what Care Programme Approach (CPA) arrangement would apply and was informed, in writing, that the CPA did not apply to such a case. No explanation was given as to why not.

With pressure from the probation service, $\mathrm{Mr}$ A was admitted as an informal patient to the local psychiatric hospital but was discharged after one week, since he had again become threatening and aggressive. He was removed from hospital, his assessment having been concluded without any involvement of probation or hostel staff, and with an assumption that he would return to the hostel. When the probation officer contacted the ward manager to discuss post-discharge arrangements, he was informed that $\mathrm{Mr} \mathrm{A}$ was not to be readmitted to the hospital and that any misbehaviour was to be seen as a relating to his "untreatable personality disorder". The social services department, by this time, had decided he was too dangerous to assess and no community care assessment was ever carried out.

In the absence of any support from health or social services and with a dangerous and deteriorating situation in the hostel, the probation officer requested Mr A's recall to prison as $\mathrm{Mr} \mathrm{A}$ was presenting a serious risk to the public. Following recall to prison, the probation officer requested the health care service to arrange a further psychiatric assessment which was carried out and $\mathrm{Mr}$ A was transferred to the local medium secure unit (MSU), within the same forensic service. The MSU declined to engage with him postrelease, and from there he was quickly transferred to a special hospital where he now remains. His prison sentence has now ended and there is no further involvement of the probation service. His ongoing care and treatment needs are now primarily the concern of health and social services.

\section{Context}

The management of $\mathrm{Mr}$ A hardly exemplifies the new direction of the Government's current mental health policy, the underlying principle of which is providing safe, sound and supportive services. It is clear from a number of government statements that new legislation is likely to be forthcoming, including a new Mental Health Act and the possibility of 'renewable detention', which will impact not only on those who suffer from mental disorder, but also on those who care for them and the wider public.

Both probation officers and psychiatrists operate in a world of changing expectations - mirrored by the concerns of politicians, the public and the press - about the way they manage difficult and often dangerous offenders with mental disorder under their care and supervision. The high-profile exposure of court cases like that of Michael Stone, or the media response to the Home Office data (1998) that in the first six months of 1997, over 100 probationers or ex-prisoners, in contact with the probation service, were charged with serious incidents, including murder or manslaughter, highlights the issue, even though these data represent only $0.1 \%$ of the total probation workload.

The work of the probation officer has already been affected by considerable legislative change starting with the Criminal Justice Act 1991, which introduced national standards, and subsequent developments that placed public protection as our primary objective.

Following the Criminal Justice Act 1991, there has been an increase in the number of offenders for whom the probation service must provide supervision after release from prison. Additionally, the Sex Offender Act 1997, and the Crime and Disorder Act 1998, will add significant numbers of serious offenders to the statutory workloads of probation staff and reflect increased involvement in public protection issues.

Under the Sex Offender Act 1997, the names and addresses of all those serving sentences for sexual offences were registered in September 1997. In future, it will become the norm for all convicted sex offenders to be registered, with their address and offending histories being known to and discussed by multi-agency risk panels. The register includes the names and addresses of sex offenders entered on local police computers. This will assist with the supervision and surveillance of convicted offenders and speed-up checks, especially on behalf of organisations responsible for the safety of children.

The Crime and Disorder Act 1998 introduces a new provision under Section 62 of the Act, allowing 
for drug treatment and testing orders, whereby the court can order a dependent drug user to submit for urine testing as well as treatment. The existence of these orders will serve to transform the way probation manages this work in association with major drug treatment agencies and local drug action teams. This also gives power to courts (Sections 5860 ) to add a period of extended post-release supervision on a person convicted of a sexual or violent offence. Supervision can be extended up to 10 years for a sex offender and up to five years for a violent offender, where the custodial term is four years or more. Given the assessed levels of mental disorder in the prison population, the probation service's new power of long-term supervision of potentially dangerous offenders will, in itself, increase the need for an improved dialogue between psychiatric services and probation.

The survey of mental disorders among prisoners issued by the Office of National Statistics for the Department of Health (Singleton et al, 1998) showed that 7 out of 10 prisoners in England and Wales were assessed as having more than one of the main types of mental disorder. The types of disorder identified included personality disorder, psychosis, neurosis, alcohol misuse and drug dependence.

The vast majority of prisoners interviewed in this vulnerable population (over $96 \%$ ) had experienced at least one stressful life event, such as running away from home, serious money problems, the breakdown of a steady relationship and the death of a close relative, and about half had experienced five or more such events. The profile of the offender referred to in our introductory case study is in no way unusual and shows the complexity of probation work with offenders with mental disorder.

A further pressure follows the 'hybrid order' arising from the Crime Sentences Act 1997. The order allows the court to pass a prison sentence on an offender and at the same time order detention in hospital for medical treatment. This order is seen as allowing the courts to deal with some of the most difficult cases in a way that takes account of the offender's need for treatment, the demands of justice and the rights of other people to be protected from harm. Also, the current concern about people with personality disorders who present genuine risk to the public but who, if they were assessed by psychiatric services, would not meet the criteria for treatment in hospital under the MHA - many of whom are not currently involved in any criminal offence - may lead to the introduction of a new form of 'renewable detention'. If this should prove to be the case, it is likely that such offenders would be detained in prison establishments despite an emphasis being made on the need to provide therapeutic interventions.
All these new measures suggest that an increasing number of offenders with mental disorder will be accessing health services through the criminal justice system and will also be the statutory responsibility of the probation service.

As can be seen, the probation service underwent a major re-framing of its purpose, increasing its statutory responsibilities and focusing the work on public protection issues as well as the more traditional role of rehabilitation. The restructuring was framed in language not dissimilar to the terminology of the Government's strategy for mental health (Department of Health, 1998). Now, for the first time in a health strategy, risk and public protection become an explicit priority, and, as with the probation service, new investment in services is to be linked to evidencebased practice - there is to be a National Service Framework with national standards, local implementation and external monitoring. Health services will have to work in partnership and demonstrate value for money. For 1999/2000, targeting better services for offenders with mental disorder will be a priority.

\section{Probation and mental disorder}

Social exclusion and deprivation have an obvious negative effect on mental health. Most of those working in psychiatric and probation services would recognise the importance of family, a home, education, employment, a cultural identity and access to health care to our sense of well-being and mental stability. Many offenders are characterised by the problems commonly associated with social deprivation including overcrowded or unsuitable accommodation, low income, unemployment, isolation and alcohol and drug misuse. Pritchard \& Cox (1993) found that of those in contact with the probation service, $33 \%$ had been in care as children; $46 \%$ had alcohol problems; $35 \%$ had drug problems; $25 \%$ had chronic housing problems; and $15 \%$ were vulnerable to human immunodeficiency virus infection.

A study of probation case loads in West Yorkshire (Ackhurst et al, 1995) showed a high rate of active suicidal ideation and recurrent histories of deliberate self-harm in the supervised offender population. The study also drew attention to the lack of current contact of this group with health services.

An internal review of the work of the ILPS indicated that about $20 \%$ of the case load showed evidence of a mental health problem. This is likely to be a significant underestimate, since the majority of probation officers reporting tend to identify offenders as having a mental disorder only where there is a formal diagnosis or some other form of corroborative 
evidence. Of those identified, one half also had significant coexisting problems of substance misuse.

Clearly, no single agency can deliver all the services required by this complex group of people. From a probation perspective, offenders with mental disorder present with a spectrum of problems, disabilities and behaviours, sometimes linked to their offending and sometimes not, but with a decided impairment on their emotional and mental health. We must, therefore, develop and enhance working partnerships with health and social services, and we need a particular alliance with psychiatry.

\section{Definition of an offender with mental disorder}

At the interface of the criminal justice and health systems, the absence of an agreed definition of what behaviours and symptoms constitute mental disorder can confuse and compound the difficulties of working together in dealing with this offender group.

Taken literally, an offender with mental disorder is someone who has broken the law and who is suffering from some form of mental vulnerability or psychological distress. The probation services in London have adopted a definition based on ICD10 (World Health Organization, 1992) as follows:

"Mentally Disordered Offenders are people who have, or are suspected of having committed an offence and who are thought to have problems generally regarded as psychiatric or psychological, whether or not they are assessed as meeting Mental Health Act criteria for inclusion in services.

Mental Disorder is a legal term within the Mental Health Act, 1983, and includes mental illness, learning disability and personality disorder.

For our purposes Mental Disorder implies the existence of a clinically recognisable set of symptoms or behaviour associated in most cases with distress and with interference with personal functions. Social deviance alone, without personal dysfunction, should not be included".

This covers a broad spectrum, which will include mental illness, personality disorder, self-harm and sexual dysfunctioning. Such a wide definition may cause difficulty to a psychiatrist working in overstretched, under-resourced services.

\section{Probation dilemmas}

Probation officers must relate to the mental and emotional health of the offender rather than concerning themselves solely with labels or clinical classifications, and they need to relate these health issues to the issue of public safety. The nature of the offence and offending history can increase the priority for interventions. They are more likely to be identifying the secondary signs of illness but need the support of psychiatry at this stage, rather than having to await the development of florid symptoms before intervention is triggered.

Probation officers have an important longitudinal perspective - having contact with offenders from the point of providing pre-sentence court reports, through supervision of community sentences and to the point of release from prison. The National Learning Set' (Home Office/Department of Health 1996) pointed out that the probation service is uniquely placed to make contact with those people, in particular young men, who are least likely to have contact with health services but who are likely to have specific mental health needs.

Probation officers need to be engaged with health and social services to ensure offenders are offered access to relevant community services. The issue for probation supervision and for the courts is that although the offender's behaviour may be subject to 'due process', the mental health of the offender also requires consideration. Criminal justice outcomes need to be appropriate to the individual's state of health and capacity to deal with the stresses of community and custodial sentences. Probation officers need to consider the offender's ability to complete any such sentence. They should also be exploring, with psychiatric services and others, opportunities for diversion from the criminal justice system and inclusion of the offender in more appropriate services.

It is important for probation and psychiatric services to develop together access to appropriate assessment of offenders with mental disorders passing through the courts, in prisons and at all other stages of contact. This will involve access to the full range of community resources including community mental health teams, community psychiatric nurses, approved social workers, and forensic psychiatric services. Likewise, there is a need to consider the role of the probation officer in the ongoing management of the offender with mental disorder once the offender is accepted into psychiatric care. There is a particular need to ensure the application of the CPA to appropriate prisoners. There must be a joint priority to tackle together the problems of prisoners who are receiving medication within prison and being released to the community with no means of continuing medication or other follow-up. Clearly, very little of this was in place for $\mathrm{Mr} \mathrm{A}$ in our case study.

The example of $\mathrm{Mr}$ A shows that even when there is a diagnosis and reporting of symptoms of major 
mental illness, from a probation perspective, there remain barriers to having an offender accepted into psychiatric services. On release from prison there can be gaps in ensuring that there are steps in place to meet the long-term community needs of someone treated for illness during their incarceration. Assertive follow-up of this group is very often required.

Probation officers continue to experience considerable difficulty in ensuring that a prisoner receiving medication within the prison has, as a minimum, the arrangements in place to access further prescriptions on release. This situation is not unique to London and was one of the issues highlighted by the Wessex Project (Lart, 1997), a Hampshire area inter-disciplinary project focused on Winchester Prison and aimed at improving access to community health services for prisoners on release, based on CPA principles.

Failing to ensure comprehensive care for the released prisoner with mental disorder can have additional unforeseen consequences. For Black people, the detection rate for psychiatric illness at primary care level is low. As a result, they are less likely to receive early interventions, and evidence shows (Pritchard \& Cox, 1998) that when they do receive attention they are more likely than their White counterparts to fall out of care. This adds to the increased likelihood of Black people being compulsorily treated and accessing that treatment through the criminal justice system. We need to ensure that once identified as in need of mental health care in the criminal justice system, Black people are not further disadvantaged by lack of follow-up on return to the community. With this group, we also need to be more mindful of the low numbers of Black people with a psychopathic disorder classification in the three English special hospitals (Ashworth, Broadmoor and Rampton) and consider the need for better screening for 'treatable personality disorder' to ensure that they are not inappropriately receiving prison sentences by default.

Professor Paul Mullen (1993) states:

"where forensic psychiatry should begin and the correctional services end, and where forensic psychiatry relinquishes domain to general psychiatry are issues debated incessantly".

Often, failure to reach a conclusion in this debate leads to a failure to provide adequate services. One of the authors was recently present at a planning conference to decide appropriate care for a young man on the cusp of child and adolescent mental health services and adult psychiatric services. The meeting had six consultant psychiatrists from a range of services. It took almost three hours of debate before one psychiatrist reluctantly accepted RMO responsibilities for the young man. This left very little time to consider and agree a proper care plan addressing not only the young man's needs, but also the very high level of risk to the public.

This conference also brought into focus other issues which impact on the delivery of care for offenders with mental disorder, for example, the degree of emphasis to be placed on treatment of the illness or on control of antisocial behaviour. The young man in question had a number of special needs, including dyslexia, and had a clear psychiatric disorder, with a diagnosis of a manicdepressive illness. He was receiving appropriate and effective medication of $800 \mathrm{mg}$ of carbamazepine daily, yet because of the high risk of his future offending, there was a suggestion that the role of the psychiatrist might be limited, with no role for mental health professionals in addressing his antisocial behaviour. The probation service's view was that there was a very direct correlation between the psychiatric disorder and the offending behaviour, and the clear outcome of not being able to produce a robust care package would be very damaging and punitive incarceration.

Probation officers often experience what they see as psychiatrists retreating from engagement with difficult and often less than pleasant individuals where multiple and overlapping problems require careful management. We see this lack of engagement as a major failure of psychiatry. This approach seems to echo a period in our history when, following publication of Martinson's 'nothing works' thesis (1974) thesis, we were dominated for 20 years by this nihilism which created the fertile grounds for Michael Howard's 'prison works' approach. Probation officers are not naïve enough to believe that all society's ills, including antisocial behaviour, can be cured by psychiatrists, but we do believe that there is a role for psychiatry in working with and advising us on the management of those offenders whose complex needs currently seem to lead to rejection by psychiatric services. Continuing relapse and re-offending is likely to be a feature of this work and should not be seen as an indicator of an offender's 'untreatability'.

\section{What do probation officers want from psychiatrists?}

Working with offenders with mental disorder can present challenges to probation officers, who are not mental health professionals, and can often be seen as further complicating working in an already 
complex criminal justice system. In working with health issues, probation officers may be working within unfamiliar structures and often they feel isolated and underskilled in this area of work. Probation officers have to practise in a context of public protection and with an outcome of reducing offending. They can only do this with the offender with mental disorder if they consider the interaction of offenders' life circumstances, the disorder that affects them and the offences they commit. Only by addressing need on these three levels can we hope to see a reduction in the risk of re-offending. The probation service could not manage this alone - indeed, no single agency can supply all the services required to meet the needs of the offender with mental disorder.

Although writing from a London perspective, where there are high levels of psychiatric morbidity and psychiatric resources are stretched, the authors are aware that across the country there is widespread and unacceptable variation in the standard of services provided for offenders with mental disorders. The proposals made below need to be in place and apply across the board, not only in London. Probation officers need to work with psychiatry to deliver good risk management, early interventions and better outreach for offenders with mental health problems if either professional group is to achieve their primary goals of public protection and care of the individual.

\section{Proposals}

There needs to be a partnership between psychiatric and probation services to agree local priorities for action, consistent with the Government's strategy for mental health. These should include development of the points discussed below (see Box 1).

\section{Inter-agency training}

Although probation officers are not mental health professionals, it is recognised (Baroness Scotland et $a l, 1998)$ that they have much to offer to the management and care of offenders with mental disorder. Training already exists for probation practitioners at pre- and post-qualification levels. Probation officers in ILPS, for example, will, after qualifying, undertake a basic training to develop their confidence in working with the medical profession and in multi-agency forums. There is also specific training for those probation officers who will work as social supervisors with conditionally discharged patients. But to take training forward,
Box 1. Action points for developing cooperation between psychiatric and probation services

Inter-disciplinary training

A joint, more consistent approach to risk assessment

Protocol for information exchange

Inclusive services for offenders with coexisting substance misuse and mental disorder

Arrangements for joint management of 'non-treatable' offenders

Effective community treatments

we need to develop inter-agency programmes where we create a shared understanding in areas such as legal issues, risk assessment and management, service provision and resources. Developing work in this area can help to overcome traditional barriers and promote the joint approach required to work across service boundaries to meet the needs of people with complex problems.

\section{A joint, more consistent approach to risk assessment}

This approach would acknowledge differences in our function and approach, but help to develop a commonly understood language and a sharing of criteria used by each discipline in their risk assessments. There is considerable scepticism about a professional's ability to predict risk accurately, and a great deal of debate about the problems of false positives. But an appropriate multi-disciplinary risk assessment carried out in a multi-agency context has a better than chance outcome in risk prediction. Such a process will allow us to identify gaps in our knowledge of people with complex problems and allow for greater corroboration of histories. We never seek to eliminate risk but to minimise it through effective management. Good risk assessment leads to better management. Defensible decision-making and a sharing of responsibility that arises out of effective interagency risk assessment also provides protection for professionals working in a potentially hazardous area.

\section{Protocols for information exchange between agencies}

In all work with offenders with mental disorder, but particularly with serious offenders who are likely 
to pose harm to the public, we need to develop agreements for the exchange of information. Relevant agencies, including health personnel, social services and police, need to be able to share concerns so that together we can define appropriate levels of service and plan appropriate action that reflects best care and enhances public protection. Confidentiality should never be used as a barrier to appropriate information exchange. Such protocols should recognise the need to assess what is to be exchanged on a case-by-case basis but within a framework of guidance agreed between our agencies. This needs to be in place, since where it does not exist, decisions to exchange information are largely left to professional judgement, and consistent practice is therefore likely to be compromised. The lack of formal guidance also leaves staff and agencies open to possible litigation and other damaging consequences arising from decisions either to exchange or not to exchange information.

\section{Inclusive services for offenders with co-existing mental disorder and substance misuse}

We should be developing joint positive approaches to offenders with complex needs. We need to guard against the tendency always to give primacy to comorbid substance misuse. The ILPS has identified that $25 \%$ of known offenders have problems related to substance misuse and also have additional mental health difficulties. Research on this group, which is currently being undertaken by ILPS, shows that when offenders not previously assumed to have mental health difficulties are stabilised on substance misuse programmes, their significant mental health problems are uncovered.

\section{Arrangements for joint manage- ment of 'non-treatable' offenders}

These arrangements are necessary for offenders who have been deemed to have a personality disorder where the condition is seen to be untreatable in terms of the MHA, and also for some sex offenders who, although not necessarily mentally ill, may need the experience and expertise of forensic psychiatry to contribute to their assessment, 'treatment' or to advise on their management.

Many in this group will be released prisoners and we need to be able to maximise use of the systems available to us, for example the CPA, ensuring delivery of the right level of support to reduce the risks of relapse and suicide. We should seek to have greater use of other community resources such as the 'probation order' with conditions of psychiatric treatment, remembering that such treatment does not need to meet MHA criteria.

\section{Effective community treatments}

These are particularly necessary for offenders identified in prison as suffering from mental disorder. We should be considering more active approaches to treatment and care, perhaps through assertive outreach. We could consider joint projects between mental health services, probation and prison services to provide continuity of care, and hopefully prevent the sort of hospital admission required by $\mathrm{Mr} A$.

\section{Conclusions}

In summary, the demands made of psychiatrists by probation officers are considerable but necessary if we are to work together in achieving better-quality health care for citizens who are also offenders, and address our common requirement to reduce risk to the public. The level of mental disorder in offender populations is high, even if the numbers with psychotic disorders is low. Studies such as the Wessex Project inform us that those offenders with mental health problems are more likely to be sentenced for violent and sexual offending. Therefore, to make progress, probation officers need psychiatrists to be available and accessible and to enter into partnership with them on the general management of complex needs. Within the framework of the Government's new strategy for mental health, it is possible that together we might be able to address some of the problems in the present system and, working in partnership, we could hope to improve the efficiency of delivery, and achieve better integration of treatment and care for offenders with mental disorder.

\section{References}

Akhurst, M., Brown, I. \& Wessely, S. (1995) Dying for Help: Offenders at Risk of Suicide. West Yorkshire Probation Services, West Yorkshire Health Authority and Association of Chief Officers of Probation (ACOP).

Baroness Scotland of Asthal QC, Kelly, H. \& Manny-Devaux, J. P. (1998) The Report of the Luke Warm Luke Mental Health Inquiry. London: Lambeth Southwark and Lewisham Health Authority.

Department of Health (1998) Modernising Mental Health Services: Safe, Sound and Supportive. London: Stationery Office.

Home Office/Department of Health (1996) Probation and Health: A Guidance Document Aimed at Promoting Effective 
Working between the Health and Probation Services. London: Stationery Office.

Home Office (1998) Serious Incident Reports: Analysis. Probation Circular 71/1998. London: Home Office.

Hudson, B. L., Cullen, C. \& Roberts, C. (1993) Training for Work with Mentally Disordered Offenders: Report of a Study of the Training Needs of Probation Officers and Social Workers. London: Central Council for Education and Training in Social Work (CCETSW).

Lart, R. (1997) Crossing Boundaries: Accessing Community Mental Health Services for Prisoners on Release. Bristol: Policy Press.

Martinson, R. (1974) What works? Questions and answers about prison reform. The Public Interest, 35, 22-53.

Mullen, P. E. (1993) Care and containment in forensic psychiatry. Criminal Behaviour and Mental Health, 3, 212225.

Pritchard C. \& Cox, M. (1998) People on Probation: Their Social Circumstances and their Characteristics (revised). Report for the King's Fund London Commission.

Singleton, N., Meltzer, H., Gatward, R., et al (1998) Psychiatric Morbidity Among Prisoners in England and Wales. London: Stationery Office.

World Health Organization (1992) ICD-10 Classification of Mental and Behavioural Disorders. Geneva: WHO.

\section{Multiple choice questions}

1. Probation officers:

a are trained mental health professionals

b consider continuing relapse and re-offending as an indicator of the untreatability of an offender with mental disorder and see no role for psychiatry with this type of offender

c need to ensure that criminal justice outcomes proposed by them to courts are appropriate to the offender's state of health

$d$ have confidence in the ability of psychiatric services to manage offenders with mental disorder with complex needs.

2. Regarding relationships between correctional and mental health services:

a the boundaries between correctional services, forensic psychiatry and general psychiatry add to the difficulties in working with complex offenders with mental health problems

b recent legislation is likely to lead to more offenders with mental disorder being diverted from the criminal justice system

c the Crime Sentences Act 1997 introduced the 'hybrid order', which allows courts to pass a custodial sentence at the same time as ordering detention in hospital for medical treatment

d post-custody arrangements for all offenders with mental disorder who were treated with medication during their incarceration are generally seen to be adequate.
3. Regarding psychiatric morbidity in offender populations:

a offenders are a particularly psychiatrically morbid group, with $70 \%$ of prisoners suffering from one or more mental disorders

b approximately $50 \%$ of all offenders with mental disoder identified in probation case loads have coexisting substance misuse problems

c studies of probation service case loads show high rates of active suicidal ideation and deliberate self-harm in supervised offenders

$\mathrm{d}$ the probation pervice is likely to have contact with those people who have mental health needs who are least likely to be in contact with health services.
MCQ answers

\begin{tabular}{|c|c|c|}
\hline 1 & 2 & 3 \\
\hline a F & a $\mathrm{T}$ & F \\
\hline b $\quad F$ & b $F$ & b $\mathrm{T}$ \\
\hline c $\quad T$ & c $\mathrm{T}$ & c $\mathrm{T}$ \\
\hline d F & d F & $T$ \\
\hline
\end{tabular}

